\title{
Constitutional Pericentric Inversion 9 in Korean Patients with Chronic Myelogenous Leukemia
}

\author{
Borum Suh, M.D. ${ }^{1}$, Jaewoo Song, M.D. ${ }^{1}$, Juwon Kim, M.D. ${ }^{1}$, Tae Sung Park, M.D. ${ }^{2}$, and Jong Rak Choi, M.D. ${ }^{1}$ \\ Department of Laboratory Medicine', Yonsei University College of Medicine, Seoul; Department of Laboratory Medicine², \\ Kyung Hee University College of Medicine, Seoul, Korea
}

\begin{abstract}
Background : Although the pericentric inversion of chromosome 9, inv(9)(p11q13), is generally considered a normal variation, it is also associated with solid tumors and several hematologic malignancies such as biphenotypic acute leukemia, ALL, AML, and myeloproliferative neoplasms. However, to the best of our knowledge, there have been no reports that suggest an association between $\mathrm{CML}$ and constitutional pericentric inversion of chromosome 9. The purpose of this retrospective study was to investigate the frequency and clinical features of CML patients with concomitant inv(9) and $t(9 ; 22)(q 34 ; q 11.2)$ variation at our institution.

Methods : We reviewed the bone marrow chromosome database entries between October 2006 and December 2008 to identify patients with concomitant inv(9) and $t(9 ; 22)$ variations. Laboratory and clinical data of the patients were obtained from the electronic medical record system.

Results : Among the $51 \mathrm{CML}$ patients, 4 (7.8\%) had concomitant inv $(9)$ and $t(9 ; 22)$ variations.

Conclusions : Although the association between inv(9) variation and CML is still controversial, we believe that hematologists should consider the role of constitutional inv(9) variation in CML patients to avoid overlooking the impaired engraftment potential of hematopoietic stem cells harboring inv(9). Therefore, we suggest that more effort should be invested to develop cytogenetic tests for detecting constitutional inv(9) variation in CML patients. (Korean J Lab Med 2010;30:218-23)
\end{abstract}

Key Words : CML, Constitutional inv(9)

\section{INTRODUCTION}

Pericentric inversion of chromosome 9, inv(9)(p11q13), is inherited as a non-pathogenic variation in normal population (0.8-2\%); however, this variation is also reported to be associated with predisposition to the development of solid tumors or acute leukemias (ALs). Keung et al. [1] reported

Received: June 10, 2009

Revision received: March 24, 2010

Accepted: April 27, 2010

Corresponding author : Jong Rak Choi, M.D.

Department of Laboratory Medicine, Yonsei University College of Medicine, 250 Seongsan-ro, Seodaemun-gu, Seoul 120-752,

Korea

Tel : +82-2-2228-2445, Fax : +82-2-313-0956

E-mail : cjr0606@yuhs.ac

ISSN 1598-6535 C The Korean Society for Laboratory Medicine that the prevalence of constitutional inv(9) variation in 799 adult AL cases was similar to that observed in the general population $(0.8 \%)$. Huh et al. [2] reported that the incidence of constitutional chromosomal aberration in hematologic malignancies was similar to that of benign hematologic disorders (2.4\% and $2.8 \%$, respectively), and the inv(9) variation was frequently observed in both groups. However, the association between constitutional inv(9) variation and CML or ALL patients with Philadelphia $(\mathrm{Ph})$ chromosome has been rarely documented [3]. To our knowledge, there have been no reports on the association between CML and constitutional pericentric $\operatorname{inv}(9)$ variation in any ethnic group. In this study, we investigated the frequency and clinical features of Korean CML patients with concomitant inv(9) and t(9;22). 


\section{MATERIALS AND METHODS}

We retrospectively reviewed the bone marrow chromosome database at the Severance Hospital of Yonsei University (Seoul, Korea) between October 2006 and December 2008. After selecting the Ph+ karyotypes, we excluded the repeated results from the same patients and identified cases with inv(9) variation among the $\mathrm{Ph}+$ cases. Clinical and laboratory data were obtained by reviewing the $\mathrm{U}$-Severance electronic medical record system.

\section{RESULTS}

Our database search revealed $103 \mathrm{Ph}+$ karyotypes. After excluding repeated results from the same patients, we identified 70 patients, including 51 patients of CML, 16 of ALL, and 3 of biphenotypic acute leukemia (BAL), with a t(9;22)(q34;q11.2) variation or BCR/ABL1 rearrangement. Among the 70 patients who underwent bone marrow and chromosome analysis, $4 \mathrm{CML}$ patients had concomitant inv(9) and $t(9 ; 22)$ variation or BCR/ABL1 rearrangement. Among the other $19 \mathrm{Ph}+\mathrm{ALL}$ or BAL patients, none had a pericentric inversion in chromosome 9 .

\section{Patient 1}

A 33-yr-old Korean man was admitted to the Severance Hospital with left upper quadrant discomfort in June 2008. The initial complete blood count (CBC) results

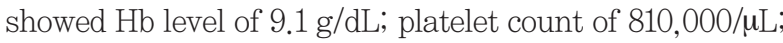
and white blood cell (WBC) count of $355,400 / \mu \mathrm{L}$ with $49 \%$ segmental neutrophils, $1 \%$ lymphocytes, $19 \%$ myelocytes, $4 \%$ metamyelocytes, $14 \%$ band forms, $4 \%$ eosinophils, $4 \%$ basophils, and 5\% immature cells. Bone marrow aspiration and biopsy analysis revealed a hypercellular marrow with a significantly large number of granulocyte precursors and megakaryocytes and a high myeloid/erythroid (M/E) ratio (30:1). The initial chromosome analysis showed the 46,XY,inv(9)(p11q13)c,t(9;22)(q34;q11.2) karyotype in all 20 metaphase cells (Fig. 1). FISH with BCR/ABL1 probe (Abbott Molecular/Vysis, Des Plaines, IL, USA) revealed the "nuc ish $(A B L 1 \times 3),(B C R \times 3),(A B L 1$ con $B C R \times 2)[91 / 252] /$ $(A B L 1 \times 2),(B C R \times 2),(A B L 1$ con $B C R \times 1)[29 / 252] /(A B L 1 \times$ $2),(B C R \times 3),(A B L 1$ con $B C R \times 2)[15 / 252]$ " karyotype, which was consistent with the abnormal pattern (1 or 2 fusions) in $53.6 \%$ of the nuclei examined. Gene rearrangement analysis was positive for $B C R / A B L 1$ (b3a2 type) (Fig. 2). The patient was receiving imatinib mesy-
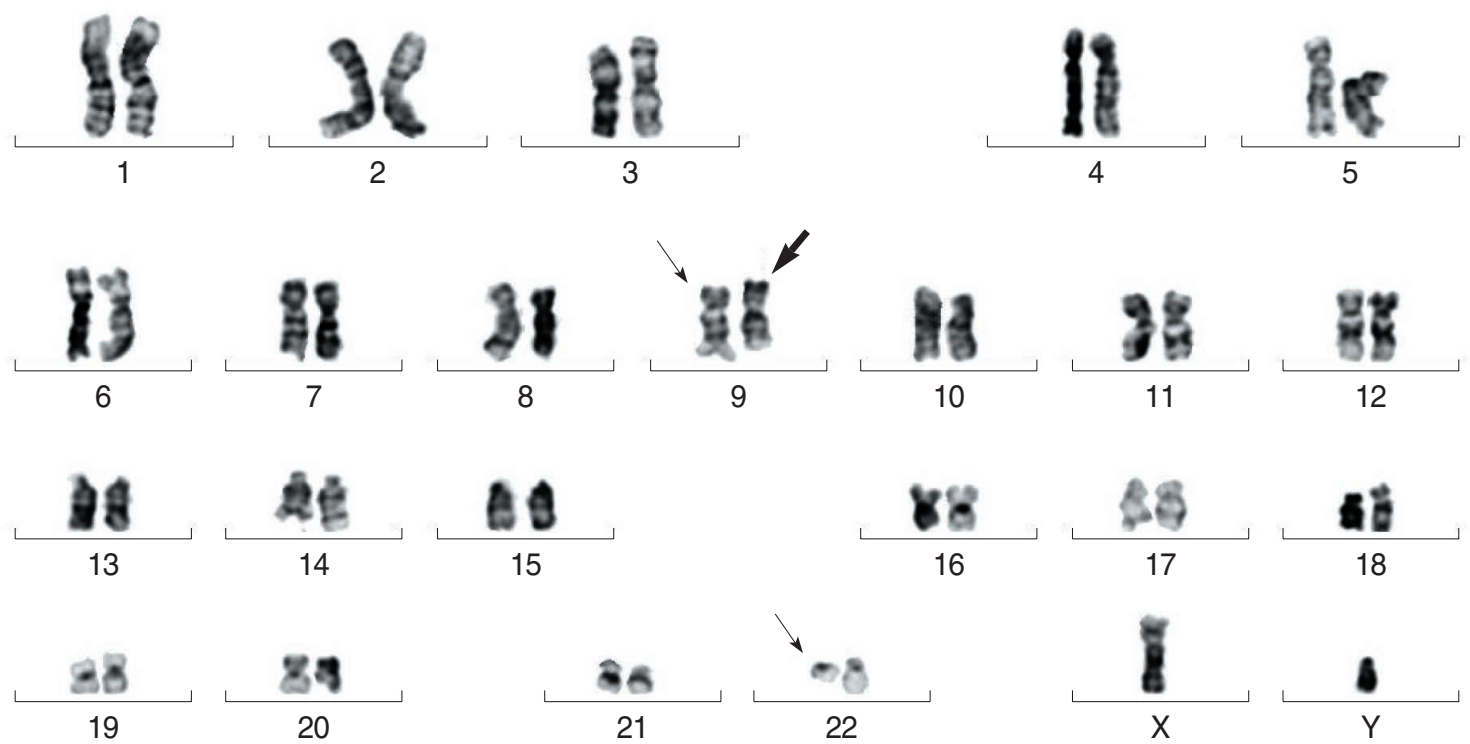

Fig. 1. The full karyogram of bone marrow cells of patient 1 is as follows: 46,XY,inv(9)(p11q13)c,t(9;22)(q34;q11.2). The bold arrow denotes $\operatorname{inv}(9)(\mathrm{p} 11 \mathrm{q} 13)$ and the single arrows denote $\mathrm{t}(9 ; 22)(\mathrm{q} 34 ; \mathrm{q} 11.2)$. 
late (IM) after initial diagnosis, but we had to stop the IM therapy after 1 month due to thrombocytopenia. His follow-up bone marrow examination revealed 20-30\% cellularity without hematologic relapse of CML. However, his second chromosome analysis still revealed the 46,XY,inv(9)(p11q13)c,t(9;22)(q34;q11.2) karyotype in 19 out of 20 analyzed cells.

\section{Patient 2}

A 64-yr-old man with a history of CML since July 2007 was brought to our hospital with fever and skin rashes for 3 days. He was first diagnosed with CML and treated with IM for 7 months. His CBC on arrival (February 2008) showed $\mathrm{Hb}$ level of $7.4 \mathrm{~g} / \mathrm{dL}$, platelet count of

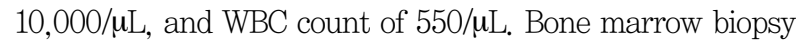
examination showed a hypocellular marrow. M/E ratio was not evaluated due to the paucity of hematopoietic cells. The karyotype of all $20 \mathrm{G}$-banded marrow metaphase cells was 46,XY,inv(9)(p11q13)c (Fig. 3). However, FISH with $B C R / A B L 1$ probe (Abbott Molecular/Vysis) revealed the "nuc ish $(A B L 1 \times 3),(B C R \times 3),(A B L 1$ con $B C R \times 2)[102 / 300] "$ karyotype, which was consistent with the abnormal signal patterns observed in $34.0 \%$ of the examined nuclei. In addition,

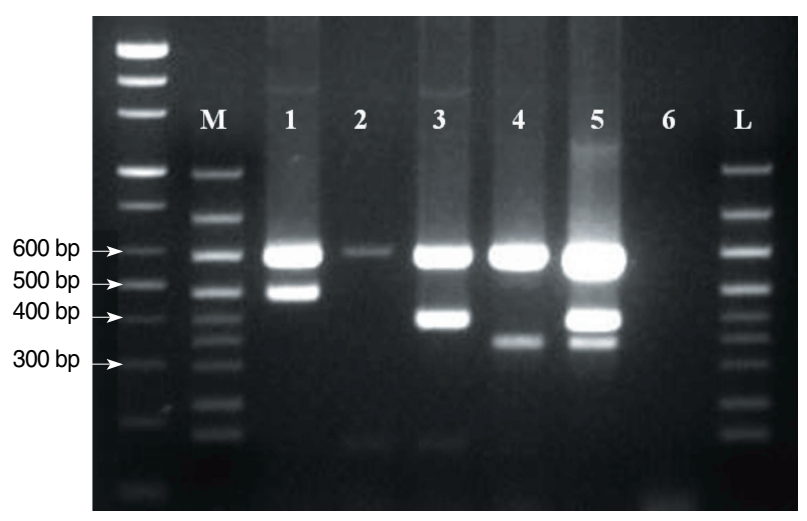

Fig. 2. Multiplex reverse transcriptase-PCR (RT-PCR) was performed using Seeplex Leukemia BCR/ABL kit (Seegene, Seoul, Korea). Lane M, molecular marker provided by manufacturer; Lane 1, sample from Patient 1 (b3a2 type); Lane 2, negative control; Lane 3, sample from Patient 3 (b2a2 type); Lane 4, positive control (e1a2 type); Lane 5, RT-PCR product of b2a2/e1a2 as a positive control; Lane 6, blank; Lane L, molecular size marker (100-bp ladder). Target and amplicon sizes are as follows: internal control (600 bp), b3a2 (476 bp), b2a2 (401 bp), and e1a2 (348 bp). gene rearrangement analysis revealed a b2a2 type $B C R / A B L 1$ rearrangement. After 1 month, he was discharged from our hospital and referred to another regional institution.

\section{Patient 3}

A 45-yr-old man was brought to our hospital with epigastric discomfort and leukocytosis in December 2008. CBC showed Hb level of $10.1 \mathrm{~g} / \mathrm{dL}$; platelet count of 397,000/ $\mu \mathrm{L}$; and WBC count of $106,700 / \mu \mathrm{L}$ with $68 \%$ segmental neutrophils, $6 \%$ lymphocytes, $5 \%$ monocytes, $8 \%$ myelocytes, $4 \%$ metamyelocytes, $8 \%$ band forms, and $1 \%$ blasts. Bone marrow aspiration showed a hypercellular marrow with a significantly large number of myeloid precursors and megakaryocytes, which was consistent with the diagnosis of CML. Chromosome analysis revealed the 46,XY,inv(9)(p11q13)c,t(9;22)(q34;q11.2) karyotype in all 20 analyzed cells (Fig. 3). The FISH analysis for BCR/ABL1 showed a "nuc ish $(A B L 1 \times 3),(B C R \times 3),(A B L 1$ con $B C R \times$ 2)[165/222]" karyotype. Gene rearrangement analysis for $B C R / A B L 1$ was positive (b2a2 type) (Fig. 2). After being diagnosed with CML in chronic phase, he was discharged

Case 2

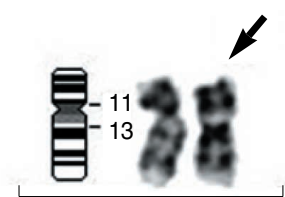

9

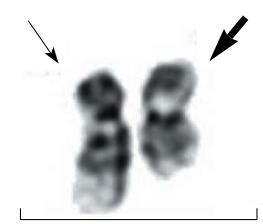

9

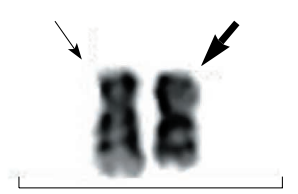

9

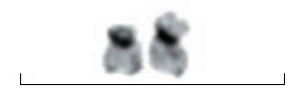

22

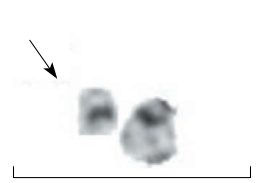

22

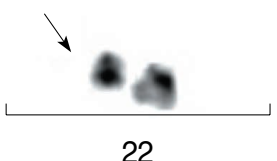

Fig. 3. The partial karyogram of bone marrow cells of patients 2 , 3 , and 4 . The bold arrows denote inv(9)(p11q13) and the single arrows denote $t(9 ; 22)(q 34 ; q 11.2)$. 
and treated with hydroxyurea on an outpatient basis.

\section{Patient 4}

A 40-yr-old woman was diagnosed with CML in chronic phase. Her chromosome analysis result in our database showed a 46,XX,t(9;22)(q34;q11.2),i(17)(q10) karyotype in 16 out of the 22 metaphase cells analyzed. However, when we reviewed our chromosome database, we detected an additional inv(9) variation in her karyogram (Fig. 3). Therefore, the revised karyotype of this patient was as follows: 46,XX,inv(9)(p11q13)c,t(9;22) (q34; q11.2),i(17)(q10)[16]/46,XX,inv(9)(p11q13)c[6]. Despite regular administration of IM, she developed neutropenic fever and septic arthritis. We suspected IM resistance because her condition deteriorated without any hematologic response and discontinued the IM therapy. HLAmatched transplantation was considered as an alternative treatment regimen.

\section{DISCUSSION}

There have been very few reports on the inv(9) variation as an acquired chromosomal abnormality in hematologic malignancies $[4,5]$, and such variations are generally regarded as a constitutional abnormality with familial inheritance. Keung et al. [1] have reported con- stitutional pericentric inversion in chromosome 9 at a frequency of $0.8-2 \%$ in normal population and at a similar frequency in AL patients. Other studies reported little difference in the incidences of constitutional chromosome aberration between patients with hematologic malignancies and the general population; however, this may either be due to the low incidence of inv(9) [6-8] or the exclusion of inv(9) [9]. Although the association between inv(9) variation and CML is still a controversial topic, constitutional pericentric inv(9) may be important for predicting impaired engraftment potential of hematopoietic stem cells harboring inv(9) [10, 11].

A careful review of literature and Mitelman database revealed that concomitant $\operatorname{inv}(9)$ and $t(9 ; 22)$ variations occurred sporadically in 8 patients [2, 3, 12-17], which included 4 CML patients, 3 ALL patients, and 1 acute basophilic leukemia patient (Table 1) [7-13]. In this study, we analyzed the data of $70 \mathrm{Ph}+$ patients diagnosed with CML, ALL, and BAL (51, 16, and 3 patients, respectively). The inv(9) variation was not detected in any Ph+ ALL or BAL patient, but it was detected at a relatively high frequency $(7.8 \%)$ in 4 out of $51 \mathrm{CML}$ patients. The detection of inv(9) is considered important in other hematologic malignancies, including ALs [1, 18, 19]. However, there have been very few studies on inv(9) detection in CML, which indicates that this variation is either overlooked or underestimated. In the fourth case in our study, we

Table 1. Reported cases of concomitant inv(9) and t(9;22) variations in $\mathrm{Ph}+$ leukemia patients

\begin{tabular}{|c|c|c|c|c|}
\hline $\begin{array}{l}\text { Case } \\
\text { No. }\end{array}$ & Sex/Age/Country & Diagnosis & Karyotypes & References \\
\hline 1 & F/55/South Africa & CML, aberrant translocation & 46,XX,inv(9)(p1?2q11),t(9;22)(p1?2;q11)/46,XX,t(9;22),i(17)(q10) & [7] \\
\hline 2 & F/35/Korea & $\mathrm{CML}$ & $47, X X, \operatorname{dup}(1)(q 21 q 44),+6, \operatorname{inv}(9)(p 11 q 12), t(9 ; 22)(q 34 ; q 11)$ & {$[8]$} \\
\hline 3 & F/55/Singapore & ALL, B-lineage & $45, X X, \operatorname{add}(7)(p 13), \operatorname{inv}(9)(p 11 q 13), t(9 ; 22)(q 34 ; q 11),-21$ & {$[9]$} \\
\hline 4 & $\mathrm{M} / 7 /$ Italy & ALL, T-lineage & $\begin{array}{l}\text { 47,Y,del(X)(q21),del(3)(q23),del(5),inv(9)(p13q13)c,t(9;22)(q34;q11), } \\
\text { add (10)(q26), +mar/47,idem,del(7)(p13) }\end{array}$ & [10] \\
\hline 5 & M/4/United States & ALL & $71, X X Y,+Y, \operatorname{inv}(9)(p 11 q 13), t(9 ; 22)(q 34 ; q 11) \times 2,+10,-16,+21$ & [11] \\
\hline 6 & F/26/United States & Acute basophilic leukemia & $46, X X, \operatorname{inv}(9)(p 11 q 13) c, t(9 ; 22)(q 34 ; q 11) / 46, X X, \operatorname{inv}(9) c, t(9 ; 22)(q 13 ; q 11)$ & [12] \\
\hline 7 & $\mathrm{M} / 24 /$ Korea & $\mathrm{CML}$ & 46,XY,inv(9)(p11q13)c,t(9;22)(q34;q11.2) & [13] \\
\hline 8 & M/52/Korea & CML & 46,XY,der(9)inv(9)(p11q13)ct(9;22)(q34;q11.2), der(22)t(9;22) & [13] \\
\hline 9 & M/33/Korea & CML & $46, X Y, \operatorname{inv}(9)(p 11 q 13) c, t(9 ; 22)(q 34 ; q 11.2)$ & Present study \\
\hline 10 & $\mathrm{M} / 64 /$ Korea & CML & $46, X Y, \operatorname{inv}(9)(p 11 q 13) c$ & Present study \\
\hline 11 & M/45/Korea & CML & $46, X Y, \operatorname{inv}(9)(p 11 q 13) c, t(9 ; 22)(q 34 ; q 11.2)$ & Present study \\
\hline 12 & F/40/Korea & CML & 46,XX,inv(9)(p11q13)c,t(9;22)(q34;q11.2),i(17)(q10) & Present study \\
\hline
\end{tabular}

Abbreviations: $\mathrm{Ph}+$, Philadelphia positive; $\mathrm{F}$, female; $\mathrm{M}$, male. 
identified a previously undetected inv(9) variation as an additional chromosomal abnormality during retrospective review. Other laboratories may similarly overlook this variation partly due to neglect of its importance, and underestimate the frequency of inv(9). In fact, in a study investigating the association between constitutional chromosomal abnormality and hematologic malignancy, the frequency of inv(9) in CML patients was observed to be $2.6 \%(2 / 77)$ [2]. The discrepancies in the frequencies may partly be due to the fact that the inv(9) variation confers an atypical chromosome morphology and complicates karyotypic analysis. Further, because the overall number of cases was limited in both the present and previous studies, the difference in frequency may not be significant [2]. More case studies are required for further elucidation of the inv(9) prevalence and clinical significance.

With rapid progress in pharmacogenetics, it is now possible to develop tailored pharmacotherapy, even for neoplastic diseases. IM has been used in tailored pharmacotherapy for the treatment of CML, clonal hypereosinophilic disorder, gastrointestinal stromal tumor, etc. Some patients develop resistance after IM therapy; however, this effect can be overcome with a standard dose or high-dose imatinib therapy in most CML patients. For patients with mutations that confer resistance to imatinib, new generation tyrosine kinase inhibitors like nilotinib and dasatinib may be effective. Thus far, no pharmacologic therapy has been effective for mutations like T315I, and stem cell transplantation is the only available treatment option.

A majority of CML patients showed significant improvement with the use of new tyrosine kinase inhibitors, but a small fraction of patients manifested multiresistance to these drugs. In such cases, additionally detected chromosomal abnormalities, whether acquired or constitutional, may partly influence the success of drug therapy or transplantation. We have suggested this possibility in the present study and our previous report [20], and emphasized the need for identifying additional chromosomal abnormalities in patients with drug resistance.
Being a retrospective analysis, the present study was limited by a small number of patients of a single ethnic group. Hence, we could not obtain data concerning further prognosis of transplantation in CML patients with inv(9). The underscoring finding of our study was that the frequency of detecting constitutional pericentric inv(9) in Korean CML patients was not lower than that in normal population. This result suggested that constitutional chromosomal abnormalities, including inv(9), should be more cautiously in CML patients of not only Korean population but of other ethnic groups as well. We think that the prevalence of constitutional inv(9) variation among CML patients is similar or slightly higher than that in the general population, although further studies in this regard are required. Therefore, on the basis of the results of our study and literature review, we propose that cytogenetic analysis should involve detection of constitutional inv(9) variation in CML patients.

\section{ACKNOWLEDGEMENTS}

The authors are grateful to Ms. HW Kil, GS Kwak, JY Kang, and KR Hong for their excellent technical support and hard work in the cytogenetic and molecular laboratory at Severance Hospital of Yonsei University Medical Center.

\section{REFERENCES}

1. Keung YK, Knovich MA, Powell BL, Buss DH, Pettenati M. Constitutional pericentric inversion of chromosome 9 and acute leukemia. Cancer Genet Cytogenet 2003;145:82-5.

2. Huh J, Chung W. Incidence and types of constitutional chromosomal abnormalities in patients with hematologic malignancies. Korean J Lab Med 2006;26:64-9.

3. Mitelman F, Johansson B, Mertens F (Eds.). Mitelman database of chromosome aberrations in cancer. http:/ / cgap.nci.nih.gov/Chromosomes/Mitelman (Updated on Nov 2008).

4. Betz JL, Behairy AS, Rabionet P, Tirtorahardjo B, Moore MW, Cotter PD. Acquired inv(9): what is its significance? Cancer Genet Cytogenet 2005;160:76-8. 
5. Wan TS, Ma SK, Chan LC. Acquired pericentric inversion of chromosome 9 in essential thrombocythemia. Hum Genet 2000;106:669-70.

6. Alimena G, Billstrom R, Casalone R, Gallo E, Mitelman F, Pasquali F. Cytogenetic pattern in leukemic cells of patients with constitutional chromosome anomalies. Cancer Genet Cytogenet 1985;16:207-18.

7. Benitez J, Valcarcel E, Ramos C, Ayuso C, Cascos AS. Frequency of constitutional chromosome alterations in patients with hematologic neoplasias. Cancer Genet Cytogenet 1987;24:345-54.

8. Cerretini R, Acevedo S, Chena C, Belli C, Larripa I, Slavutsky I. Evaluation of constitutional chromosome aberrations in hematologic disorders. Cancer Genet Cytogenet 2002;134:133-7.

9. Welborn J. Constitutional chromosome aberrations as pathogenetic events in hematologic malignancies. Cancer Genet Cytogenet 2004; 149:137-53.

10. Imashuku S, Naya M, An B, Nakabayashi Y, Kuriyama K, Udeda I, et al. Constitutional pericentric inversion of chromosome 9 and haemopoietic stem cell transplantation: delayed engraftment. Br J Haematol 2002;118:1195-6.

11. Keung YK, Knovich MA, Hurd DD, Pettenati M. Constitutional pericentric inversion of chromosome 9 and bone marrow transplantation. Br J Haematol 2003;123:748-9.

12. Bernstein R, Pinto MR, Wallace C, Penfold G, Mendelow B. The incidence, type, and subsequent evolution of 14 variant Ph1 translocations in 180 South African patients with Ph1-positive chronic myeloid leukemia. Cancer Genet Cytogenet 1984;12:225-38.

13. Lee DS, Lee YS, Yun YS, Kim YR, Jeong SS, Lee YK, et al. A study on the incidence of $A B L$ gene deletion on derivative chromosome 9 in chronic myelogenous leukemia by interphase fluorescence in situ hybridization and its association with disease progression. Genes Chromosomes Cancer 2003;37:291-9.

14. Lim LC, Heng KK, Vellupillai M, Tan LT, Boey BC, Lau LC, et al. Molecular and phenotypic spectrum of de novo Philadelphia positive acute leukemia. Int J Mol Med 1999;4:665-7.

15. Lo Nigro L, Sainati L, Mirabile E, Lanciotti M, Poli A, Leszl A, et al. Association of cytogenetic abnormalities with detection of BCR$A B L$ fusion transcripts in children with T-lineage lymphoproliferative diseases (T-ALL and T-NHL). Pediatr Blood Cancer 2004;42: 278-80.

16. Ohtaki K, Abe R, Tebbi CK, de los Santos R, Han T, Sandberg AA. Near-triploid Ph-positive leukemia. Cancer Genet Cytogenet 1985; 18:113-21.

17. Peterson LC, Parkin JL, Arthur DC, Brunning RD. Acute basophilic leukemia. A clinical, morphologic, and cytogenetic study of eight cases. Am J Clin Pathol 1991;96:160-70.

18. Lourenco GJ, Silva PM, Bognone RA, De Souza RA, Delamain MT, Lima CS. Inherited pericentric inversion of chromosome 9 in acquired hematological disorders. Ann Hematol 2007;86:465-7.

19. Manola KN, Harhalakis N, Symeonidis A, Rigana H, Stavropoulou C, Karakasis D, et al. Constitutional pericentric inversion of chromosome 9 and hematopoietic recovery after allogeneic stem cell transplantation. Ann Hematol 2006;85:611-5.

20. Park TS, Cheong JW, Kim SJ, Lee KW, Song J, Lee KA, et al. Concomitant $\mathrm{t}(3 ; 3)(\mathrm{q} 21 ; \mathrm{q} 26)$, trisomy 19, and E255V mutation associated with imatinib mesylate resistance in chronic myelogenous leukemia. Cancer Genet Cytogenet 2009;190:46-8. 